\title{
KAON ELECTROPRODUCTION AT LARGE MOMENTUM TRANSFER
}

\author{
PETE E.C. MARKOWITZ \\ For the Jefferson Lab Hall A and E98-108 Collaborations \\ Physics Department \\ Florida Intemational University \\ Miami, FL USA \\ E-mail: markowit@fiu.edu
}

Exclusive $\mathrm{H}\left(\mathrm{e}, \mathrm{e}^{\prime} \mathrm{K}\right) \mathrm{Y}$ data were taken in January, March and April of 2001 at the Jefferson Lab Hall $A$. The electrons and kaons were detected in coincidence in the hall's two High Resolution Spectrometers (HRS). The kaon arm of the pair had been specially outfitted with two aerogel Cerenkov threshold detectors, designed to separately provide pion and proton particle identification thus allowing kaon identification. The data show the cross section's dependence on the invariant mass, $W$, and 4-momentum transfer, $Q^{2}$, along with results of systematic studies. Ultimately the data will be used to perform a Rosenbluth Separation as well, separating, the longitudinal from the transverse response functions. Preliminary data on this $\mathrm{L} / \mathrm{T}$ ratio are presented.

\section{Introduction}

Exploring the electromagnetic structure of the hadronic spectrum is part of Jefferson Lab's primary mission of basic research into the nuclear building blocks. The kinematical region accessible with beam energies upto $6 \mathrm{GeV}$ allows investigation of both nucleon and light meson structure.

The electromagnetic production of kaons allows measurement of the structure of mesons containing a strange quark. Jefferson Lab operates in an ideal kinematical range for such studies; Jefferson Lab is able to measure strange quark electroproduction from threshold through the deep-inelastic scattering (DIS) region.

Experiment E98-108 ${ }^{1}$ was approved to measure kaon electroproduction over a broad kinematical range. The experiment separates the longitudinal, transverse, and longitudinal-transverse interference responses to the unpolarized cross section. One goal is to obtain a data set allowing the extrapolation (in the Mandelstam variable $t$ ) of the isolated longitudinal response to the kaon mass pole. The reaction at the kaon mass pole would correspond to scattering an electron off of a free kaon and is correspondingly sensitive to the internal elecromagnetic structure of the kaon. By extrapolating the data taken to the mass pole, the goal is to constrain that kaon electromagnetic form factor. 
A second goal is to examine the behavior of transverse response in this kinematical region, which overlaps with both the resonance region and extends DIS region. The kaon production reaction been calculated in terms of the hard scattering model of Brodsky and Lepage with three different baryon Distribution Amplitudes ${ }^{2}$. A quark calculation to leading twist using the Born approximation is also available ${ }^{3}$. Both calculations are for photoproduction; similar electroproduction calculations for the transverse response are not available.

A third goal is to use the longitudinal-transverse interference response function to constrain which reaction models contribute to the measurement. Once these reaction models are understood, the longitudinal response will provide sensitivity to the kaon electromagnetic form factor.

\section{Present Status}

Measurements of the $\gamma+p \rightarrow K^{+}+Y$ and $e+p \rightarrow e^{\prime}+K^{+}+Y\left(Y=\Lambda, \Sigma^{0}\right)$ reactions are limited by short lifetimes $\left(c \cdot t_{K}=370 \mathrm{~cm}, c \cdot t_{\Lambda}=8 \mathrm{~cm}\right)$, small production rates (an order of magnitude smaller than for pions) and high thresholds $\left[E_{t h}(K \Lambda)=911 \mathrm{MeV}, E_{t h}\left(K \Sigma^{0}\right)=1.05 \mathrm{GeV}\right]$. The unseparated cross sections are known with an accuracy of about $10 \%^{11,9,12}$ and provide an empirical fit to the unseparated cross section based on phase space, a simple monopole $Q^{2}$-form factor, and an exponential drop with $t$. The past ten years have seen the first new data on electromagnetic kaon production.,5

Recent data on kaon photoproduction shows a more complicated relation between the coupling of resonances and the kaon-hyperon final state system. The single polarization asymmetries are available for $\Lambda$ production with errors of $25 \%$ to $50 \%$. The photon energy range in the published data is limited to $0.9 \leq E_{\gamma} \leq 1.4 \mathrm{GeV}$ (a few additional points were measured ${ }^{6,4}$ at a fixed momentum transfer $t=-0.147 \mathrm{GeV}^{2}$ in the energy range $E_{\gamma}=1.05-2.2$ $\mathrm{GeV}$ ). On the theoretical side, new calculations based on Regge models now provide fits comparable to the hadrodynamic models which include higher spin resonances to fit the photoproduction data. ${ }^{7,8}$ )

However the current situation for kaon electroproduction remains less satisfactory, both from the experimental and theoretical point of view. Jefferson Lab experiment E93-108 ${ }^{5}$ was the first actual Rosenbluth separation, and demonstrated that the longitudinal response is large (approximately three quarters the size of the transverse response) at $t=t_{\min }$ or $\theta_{c m}=0$. That experiment also demonstrated that Jefferson Lab is well suited for such precision separations, with the small emittance of the beam, the excellent particle 
identification of the detectors, and the accuracy of the spectrometers for cross section measurements.

In kaon electroproduction, the mass pole is in the unphysical region where $t>0$. Instead, the electroproduction process attempts to isolate the $t$-channel by extrapolating the longitudinal response in $t$ to the unphysical kaon mass pole. Such an extrapolation is model dependent meaning that it is desirable to check the extrapolation against available data on the kaon form factor at lower $Q^{2}$.

The only unambigous data on the kaon form factor is from high-energy scattering of kaon beams from atomic electrons. ${ }^{13}$ Due to the inverse kinematics, the measurements were limited to low 4-momentum transfers $(.02<$ $\left.Q^{2}<.12(\mathrm{Gev} / \mathrm{c})^{2}\right)$. The measurements were able to determine the kaon charge radius by looking at the slope of the cross section with respect to $Q^{2}$. [Interestingly, within the next year the addition of the septum magnets to Hall A would let Hall A do a comparison of electroproduction with actual meson-electron elastic scattering in about 3 days for the kaon, plus about the same for the pion.]

\section{Experimental Setup}

The experiment took place in Hall $A$ at the Thomas Jefferson National Accelerator Facility's CEBAF accelerator. The standard equipment in Hall $A$ has been described elsewhere. ${ }^{14}$ Electron beams of energies upto $5.7 \mathrm{GeV}$ were incident on liquid hydrogen targets of nominal $4 \mathrm{~cm}$ and $15 \mathrm{~cm}$ lengths. Electrons and kaons were detected in coincidence in the two magnetically symmetric high resolution spectrometers (HRS).

The HRS spectrometers were outfitted with special particle identification: on the electron side there were a gas Cerenkov counter and a lead glass calorimeter to veto $\pi^{-}$mesons, while on the hadron side two different aerogel Cerenkov counters were used to separately veto pions and protons.

The pions were vetoed in the Al aerogel which has a refractive index of 1.015 by requiring that the detector A1 not fire. Protons were vetoed in the A2 aerogel which has a refractive index of 1.055 by requiring that the detector did fire.

\section{Preliminary Results}

Figure 1 shows the reconstructed mass of the unobserved baryon in the $H\left(e, e^{\prime}\right.$ K) reaction, in this case either a $\Lambda$ or $\Sigma$ hyperon. The ratio of these two production cross sections is observed to drop rapidly with $Q^{2}$, however because 


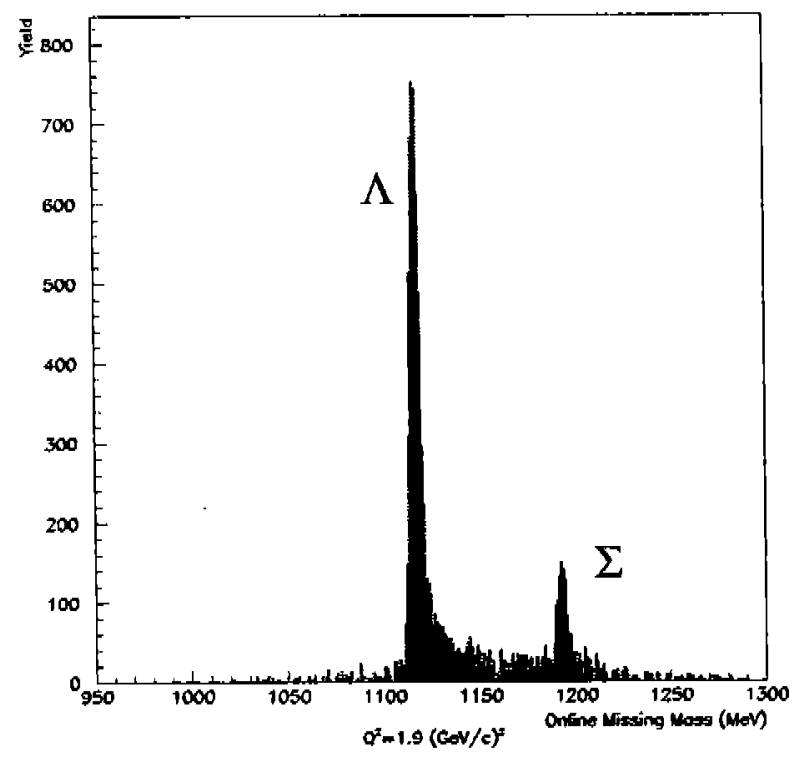

Figure 1. The $\Lambda$-hyperon reconstructed from the $\left(\mathrm{H}\left(e, \mathrm{e}^{\prime} \mathrm{K}\right) \mathrm{Y}\right.$ missing mass.

the phase space acceptance for the two reactions also changes rapidly, final results are dependent on the ongoing acceptance studies. The quality of the particle identification can be judged by the lack of background in the plot.

The range in $W$ covered by the experiment does not show any striking behavior; the unseparated cross sections smoothly follows the nearly flat reaction phase space as shown by the photoproduction data. Similarly, the unseparated cross sections drop off approximately as $1 /\left(Q^{2}+2.67\right)^{2}$ as given by the E93-108 data. Here minor discrepancies to the global fit should be expected since the data were all taken at different $\varepsilon$-values (the virtual photon polarization).

Figure 2 shows the very preliminary separations for the data at $Q^{2}=2.4$ $(\mathrm{GeV} / \mathrm{c})^{2}$ and $W=1.8,1.9 \mathrm{GeV}$ plotted versus $\varepsilon$, the polarization of the virtual photon. A straight line fit to those data will provide the separated longitudinal and transverse contributions. The inner errror bars are the statistical errors while the outer error bars reflect a conservative estimate of what the systematic uncertainties are at this point in the analysis. The ultimate 

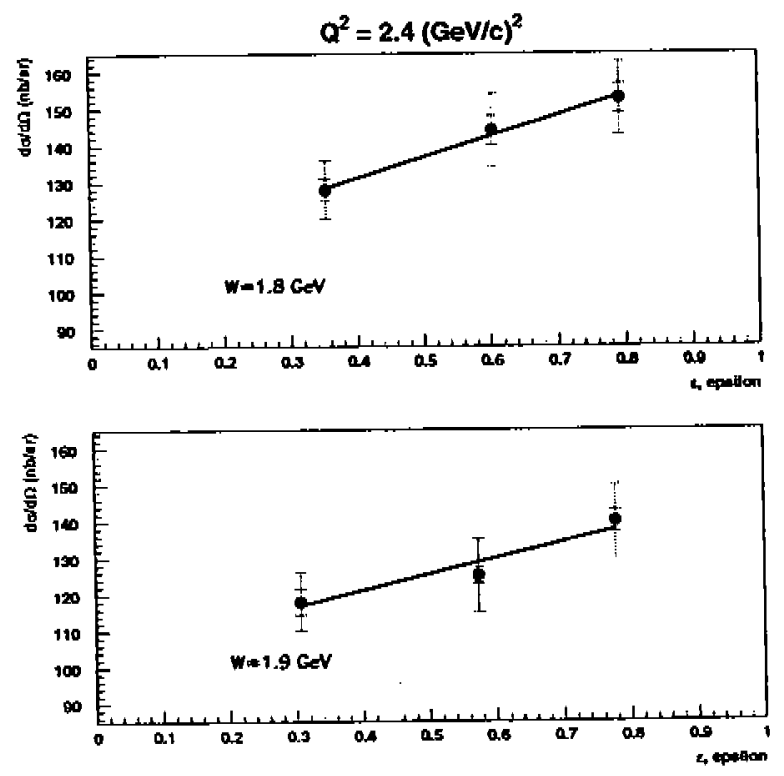

Figure 2. The cross sections at $Q^{2}=2.4(\mathrm{GeV} / \mathrm{c})^{2}$ and $W=1.8,1.9 \mathrm{GeV}$ plotted versus $\varepsilon$.

error bars will be dominated by the statistics. As can be seen from the plot, at the larger momentum transfers of this experiment (as compared to E93-018), the longitudinal cross section is decreasing relative to the transverse but is still appreciable.

\section{Outlook}

The data analysis is continuing. The preliminary results indicate that the quality of the data is excellent and show that the longitudinal response decreases faster than the transverse with increasing $Q^{2}$. Additonal results are expected in the coming months.

The collaboration is finishing construction of two septum magnets which will be used with nuclear targets for hypernuclear production experiments. The resolution is critical for hypernuclear measurements making the Hall A HRS a good match. The backgrounds are also expected to rise in these exper- 
iments, and the collaboration, led by the Roma'/INFN group is constructing a RICH to provide additional particle identification. We expect to run the hypernuclear experiment in the fall of 2002.

At higher energies there is the possiblity of looking at semi-inclusive production off polarized targets to measure the sea quark distributions as well; such data would be complementary to the existing Drell-Yan and Hermes data.

\section{References}

1. Jefferson Lab Experiment E98-108, P. Markowitz, M. Iodice, S. Frullani, C. C. Chang, O. K. Baker spokespersons (1998).

2. P. Kroll, M. Schurmann, K. Passek, W. Schweiger, Phys. Rev. D $\underline{5} 54315$ (1997).

3. G. R. Farrar, K. Huleihel, H. Zhang, Nucl. Phys. B349, 655 (1991).

4. M. Bockhurst etal., Z. Phys. C $\underline{63} 37$ (1994).

5. G. Niculescu, etal., Phys. Rev. Lett. $\underline{81}, 1805$ (1998).

6. P. Feller, et.al., Nucl. Phys. B39 413 (1979).

7. J.C. David, C. Fayard, G.H. Lamot, B. Saghai, Phys. Rev. C $\underline{53} 2613$ (1996).

8. R. A. Adelseck, B. Saghai, Phys. Rev. C $\underline{42} 108$ (1990).

9. C. J. Bebek et al., Phys. Rev. D $\underline{15}, 47$ (1977).

10. T. Azemoon et al., Advance in Nucl. Phys. 17, 47 (1987).

11. P. Brauel et al., Z. Physik, $\underline{3}, 101$ (1979).

12. A. Bodek et al., Phys. Lett. $\underline{51 B}, 417$ (1974).

13. S. R. Amendolia et.al., Phys. Lett. B 178, 435 (1986).

14. The Jefferson Lab Hall A Collaboration, Paper in progress, to be submitted to Nuclear Methods and Instrumentation. 